\title{
Journal of Real-Time Image Processing: second issue of volume 8
}

\author{
M. F. Carlsohn $\cdot$ N. Kehtarnavaz
}

Published online: 7 May 2013

(c) Springer-Verlag Berlin Heidelberg 2013

The editorial board of JRTIP met in San Francisco in February where they were attending the 2013 SPIE Conference on Real-Time Image and Video Processing. A picture of some of the editorial board members present at the Springer sponsored dinner meeting is shown below. The editorial board will have its next meeting in Brussels in April 2014 where the SPIE Conference on Real-Time Image and Video Processing will take place as part of the SPIE Photonics Europe.

In the recently held editorial meeting, the following issues were discussed which are worth noting here:

Last year in 2012 more than 350 manuscripts were submitted to JRTIP, which was a substantial increase over the previous year in 2011. Although this is an indication that the recognition of JRTIP is steadily increasing, it has also created a challenge in terms of timely reviews of manuscripts.

(a) So far the review load per AE has been kept to four manuscripts per year on average. However, it was acknowledged that this model was no longer sustainable and it was necessary to increase the review load to six manuscripts per year to be able to cope with the large number of manuscripts that are being submitted to JRTIP.

(b) Two recommendations came out of the meeting addressing how to find quality reviewers who would be more likely to accept invitations to review

\section{F. Carlsohn ( $\bowtie)$}

Engineering and Consultancy for Computer Vision and Image

Communication, Bremen, Germany

e-mail: Matthias.Carlsohn@t-online.de

N. Kehtarnavaz

University of Texas at Dallas, Dallas, TX, USA

e-mail: kehtar@utdallas.edu manuscripts. Besides personal contacts, the following two sources were noted to be effective ways of achieving this objective:

(b.1) selection from authors who have previously published in JRTIP in similar areas

(b.2) selection from the reference list of a submitted manuscript.

Normally, there is a good chance researches identified as such would agree to act as reviewers since they do similar research work and the manuscript would be of interest to them.

(c) In addition to the JRTIP successful reviewer reward program enacted last year, more information at http://www.springer.com/11554, reviewers having performed $10+$ high quality reviews for JRTIP are encouraged to join the editorial board as Associate Editors by offering special issues of their interests.

This regular issue includes six papers. The first paper by Deforges et al. presents a computationally efficient algorithm for performing morphological operations based on a recursive morphological decomposition method of eightconvex structuring elements. This approach is shown to achieve a low computational complexity. The second paper by Vytla et al. presents a real-time hardware implementation of a gradient domain dynamic range compression algorithm for high dynamic range images. The hardware implementation of the developed algorithm is described in VHDL and synthesized for an FPGA device achieving a rate of about 100 frames per second. The third paper by Maurice et al. presents a coded structured light for 3D reconstruction of poorly or non-textured scenes. It is shown that due to a mixed exploration/exploitation search 
behaviour and a $\mathrm{O}\left(n^{2}\right)$ to $\mathrm{O}(n)$ complexity reduction using the epipolar constraint, the real-time coding of patterns can be achieved. The fourth paper by Esteghamatian et al. presents a three-stage framework for real-time $2 \mathrm{D}$ into $3 \mathrm{D}$ cardiac image registration during respiration, which is composed of prior registration to extract the trend of respiratory motion and to calibrate a set of time series predictors for future motion prediction. The fifth paper by Schatz describes a hardware implementation of the contrast-limited adaptive histogram equalization algorithm for processing of image sequences from high-dynamic-range infrared cameras. The resource usage of the histogram equalization module and how it depends on its parameters has been determined and the design has been synthesized and tested on a Xilinx FPGA. The sixth and final paper by Bagherinia et al. describes a fast algorithm to detect special multi-colour markers with a camera cell phone. Robust detection is achieved at a very low computational cost using a cascade of elemental detectors.

At the end, as always we are grateful to the associate editors and reviewers for their volunteering efforts in handling the review processes and providing quality reviews as well as to the guest editors who are currently offering several special issues with the call for papers appearing at the end of this issue.

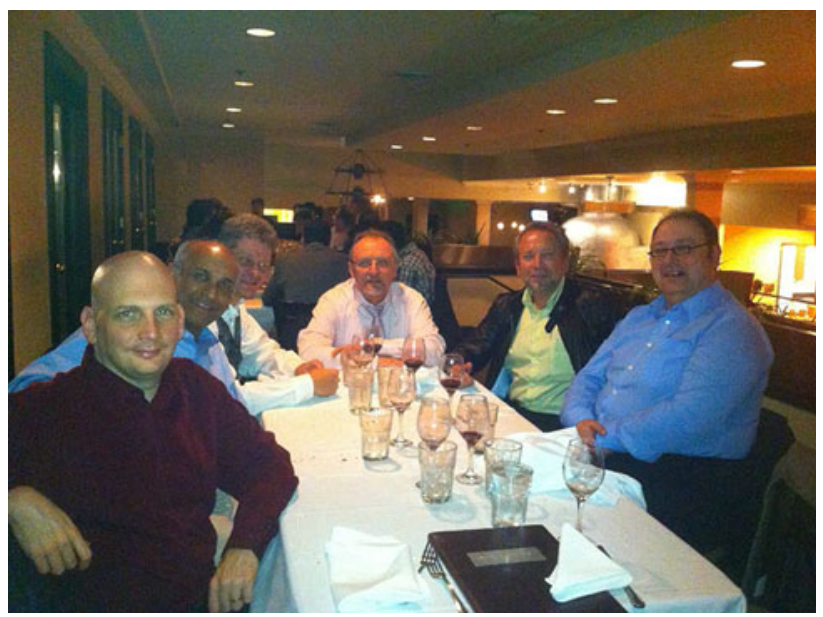

From left to right: Barak Fishbain, Mukul Shirvaikar, Sergio Goma, Matthias Carlsohn, Volodymyr Ponomaryov, Christos Grecos, Nasser Kehtarnavaz (taking picture) 